\section{Treatment burden}

\author{
Lisa A. Harvey ${ }^{1}$ - Gunilla M. Åhrén ${ }^{2,3}$
}

๑) International Spinal Cord Society 2019

Have you ever wondered how many hours per day are required by people with spinal cord injuries (SCI) to comply with all the recommendations made by the various health professionals involved with their ongoing care? Or how much money and time it would cost to follow all of the health professionals' advice? A study recently looked at the time, money and effort required by people with any three out of six chronic conditions (not SCI) to comply with key guidelines. It found that to comply with guidelines these people would need to:

“.... take from six to 13 different drugs a day, visit a health professional 1.2 to 5.9 times a month, and spend a mean (SD) of 49.6 (27.3) to 71.0 (34.5) hours each month in health related activities" $[1$, p. 1].

Presumably, some people with SCI would need to devote even more time than this if they were to comply with the various recommendations of all healthcare professionals. This has the potential to lead to treatment burden.

Treatment burden for people with SCI may be due to two main reasons. Firstly, healthcare professionals tend to focus on outcomes without carefully considering the acceptability and feasibility of the treatment to the person with SCI (a concept similar to that discussed in a recent editorial on the minimally worthwhile treatment effect) [2]. The second reason why people with SCI are often asked to devote unreasonable amounts of time to their health is because there is a lack of coordination between various healthcare professionals and a tendency for each of them to only focus on what they understand best, namely their area of expertise. So pamphlets, books and websites providing advice are put together without sufficient input from all the various healthcare professionals

Lisa A. Harvey

spinalcord@iscos.org.uk

1 University of Sydney, Sydney, Australia involved in overall care, and most importantly, without sufficient input from those living with SCI. Each healthcare professional thinks about his/her small area of expertise. This seems perfectly reasonable; however, it is not reasonable if all the bits of advice add up to an unreasonable expectation put on the person with SCI.

Of course, these issues are not simple to resolve because by necessity there are many different things that a person with SCI could (and sometimes, should) do to stay fit and healthy. However, unless the advice provided to people with SCI takes into account the overall treatment burden, people with SCI will intuitively or explicitly prioritise for themselves what they focus on (or be limited by lack of time, money, and access to health care). This is to be expected, but it may be preferable to do this in consultation with healthcare professionals, who are sympathetic to the problems of healthcare burden, so as to ensure treatment priorities are based on evidence and clinical experience, as well as the lived experience of people with SCI.

Spinal Cord welcomes research that investigates treatment burden. There are at least two outcome measures that are used for this purpose in other areas of medicine which may be appropriate for our field. They are the Treatment Burden Questionnaire (TBQ) [3] and the Patient Experience with Treatment and Self-management (PETS) [4]. With a better understanding of treatment burden, healthcare professionals may be better positioned to partner with people with SCI to help them decide which health-care recommendations they should prioritise.

\section{Compliance with ethical standards}

Conflict of interest The authors declare that they have no conflict of interest.

2 The Gothenburg Competence Centre for Spinal Cord Injury, Gothenburg, Sweden

3 European Spinal Cord Injury Federation, Nottwil, Switzerland 
Publisher's note: Springer Nature remains neutral with regard to jurisdictional claims in published maps and institutional affiliations.

\section{References}

1. Dobler CC, Harb N, Maguire CA, Armour CL, Coleman C, Murad MH. Treatment burden should be included in clinical practice guidelines. BMJ. 2018;363:k4065.
2. Harvey LA. A minimally important treatment effect is a key but elusive concept. Spinal Cord. 2019;57:83-4.

3. Tran V-T, Harrington M, Montori VM, Barnes C, Wicks P, Ravaud P. Adaptation and validation of the Treatment Burden Questionnaire (TBQ) in English using an internet platform. BMC Med. 2014;12:109.

4. Eton DT, Yost KJ, Lai JS, Ridgeway JL, Egginton JS, Rosedahl $\mathrm{JK}$, et al. Development and validation of the Patient Experience with Treatment and Self-management (PETS): a patient-reported measure of treatment burden. Qual Life Res. 2017;26:489-503. 